\title{
AUTOMATIC AND GENERIC MOSAICING OF MULTISENSOR IMAGES: AN APPLICATION TO PLEIADES HR
}

\author{
F. Bignalet-Cazalet ${ }^{\text {a, } *, \text { S. Baillarin }}{ }^{\text {a }}$ C. Panem ${ }^{\text {a }}$ \\ ${ }^{a}$ Centre National d'Etudes Spatiales, 10 avenue Belin, 31400 Toulouse, FRANCE \\ (francois.bignalet-cazalet, simon.baillarin, chantal.panem)@cnes.fr
}

KEY WORDS: Image processing, Registration, Mosaic, Pleiades.

\begin{abstract}
:
In the early phase of the Pleiades program, the CNES (the French Space Agency) specified and developed a fully automatic mosaicing processing unit, in order to generate satellite image mosaics under operational conditions. This tool can automatically put each input image in a common geometry, homogenize the radiometry, and generate orthomosaics using stitching lines.

As the image quality commissioning phase of Pleiades1A is on-going, this mosaicing process is being tested for the first time under operational conditions. The French newly launched high resolution satellite can acquire adjacent images for French Civil and Defense User Ground Segments. This paper presents the very firsts results of mosaicing Pleiades1A images.

Beyond Pleiades' use, our mosaicing tool can process a significant variety of images, including other satellites and airborne acquisitions, using automatically-taken or external ground control points, offering time-based image superposition, and more. This paper also presents the design of the mosaicing tool and describes the processing workflow and the additional capabilities and applications.
\end{abstract}

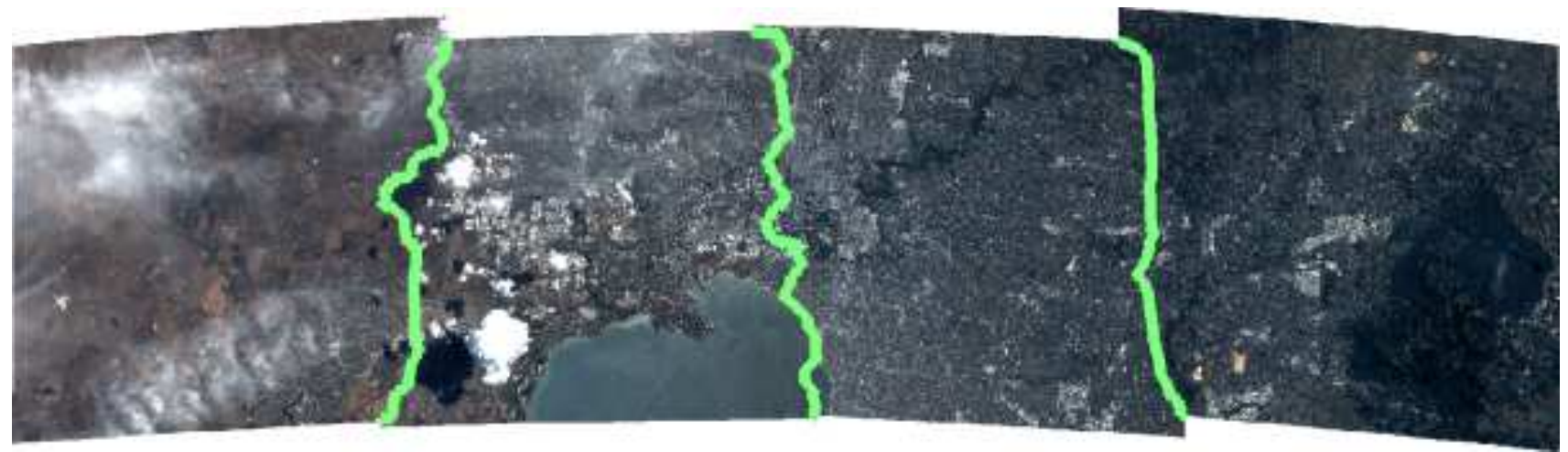

Figure 4. Pleiades' 4 strips mosaic of Melbourne with stiching line @CNES Copyright 2012

\section{INTRODUCTION}

SIGMA is the tool, designed and developed by CNES (Centre National d'Etudes Spatiales, the French Space Agency), for the automatic generation of satellite image mosaics. It automatically homogenizes the radiometry and the geometry of unitary input strips, and generates orthomosaics.

SIGMA was designed and developed for the Pleiades satellites operational Image Processing Unit. Pleiades High Resolution, the French Civil and Defense two-satellite Earth Observation constellation, acquires images with a resolution of $70 \mathrm{~cm}$ (in nadir condition for panchromatic band) with a swath of $20 \mathrm{~km}$. A larger swath can then be obtained using the platform high agility to acquire adjacent image strips (Fig. 3) together with the automatic image processing on ground. Hence, the system is able to offer to end user $60 \mathrm{~km} * 60 \mathrm{~km}$ (and more) images from $20 \mathrm{~km}$ strips.
With the successful launched of Pleiades1A on 2011 December 16th, this full mosaicing process is now used in the Pleiades operational Image Processing Unit. During the image quality commissioning phase, it will be tested and optimized to ensure its image quality and operational performances. Each step (spectral band registration, registration within input images, registration with external references, radiometric harmonization, stitching line computation and mosaic resampling) has to be tuned according to the real satellite configuration 


\section{PLEIADES 1A IMAGES}

\subsection{The Pleiades system}

The PLEIADES system is an optical observation system with submetric resolution designed to satisfy both civilian and military requirements.

The space component is made of two "small satellites" (mass of one ton). Their great agility enables daily access all over the world, which is a critical need for defense and civil security applications, and a coverage capacity necessary for cartography applications at scales better than those accessible to the SPOT family satellites. To achieve this, the Pleiades constellation is placed on a Sun-synchronous orbit at $694 \mathrm{~km}$. The first satellite, Pleiades1A was launched by Soyuz from Europe's spaceport in Kourou, French Guiana, on December, 16th, 2011 and the second one, Pleiades 1B will be launched by the end of 2012 .

The ground segment allows military or civilian users to submit requests, to receive the image telemetry and to process to obtain the desire final product. In particular, users can choose a Region Of Interest greater than $20 \mathrm{~km}$ (a swath). In this case, the system has to deliver a mosaic-level product, automatically generated and elaborated with the adjoining products.

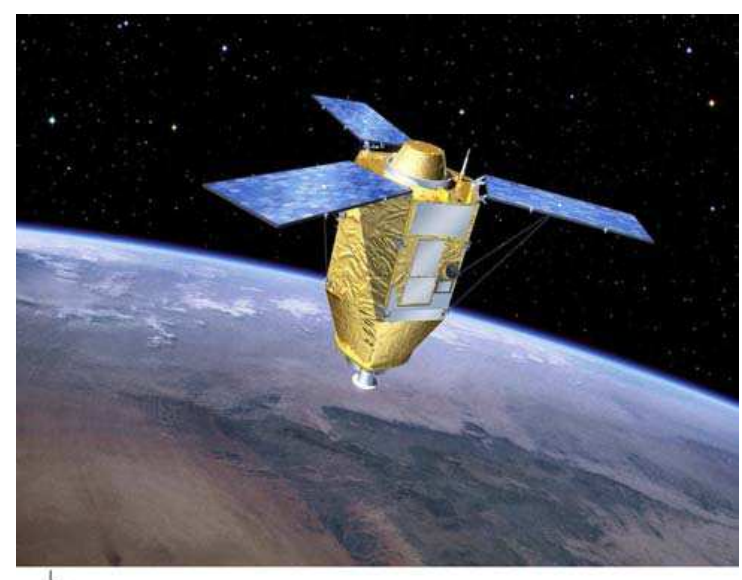

(b)

Figure 2. Pleiades 1A Satellite (Artist's view)

\subsection{The Pleiades mosaics}

Three days after launch, the first Pleiades1A image was produced by the Image Production Unit. Since December 20th, the image quality commissioning phase has been on-going. The mosaicing process is being validated and tuned.

The Pleiades operational Image Processing Unit, in the main civilian centre (the Astrium Geo Information centre in Toulouse), can easily process a dozen of mosaics per day, in addition to other product levels such as Perfect Sensor or Orthoimages. A mosaic production takes less than 3 hours on the Pleiades civil IPU for a $60 * 60 \mathrm{~km}^{2}$ mosaic (less than $20 \mathrm{~min}$ per $20 * 20 \mathrm{~km}^{2}$ image)

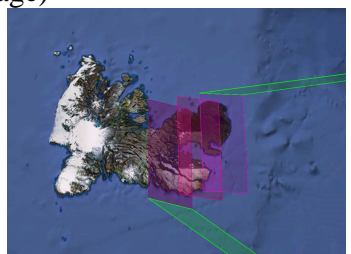

Figure 3. Illustration of Pleiades' adjacent acquisitions of Kergelen Island with GoogleEarth

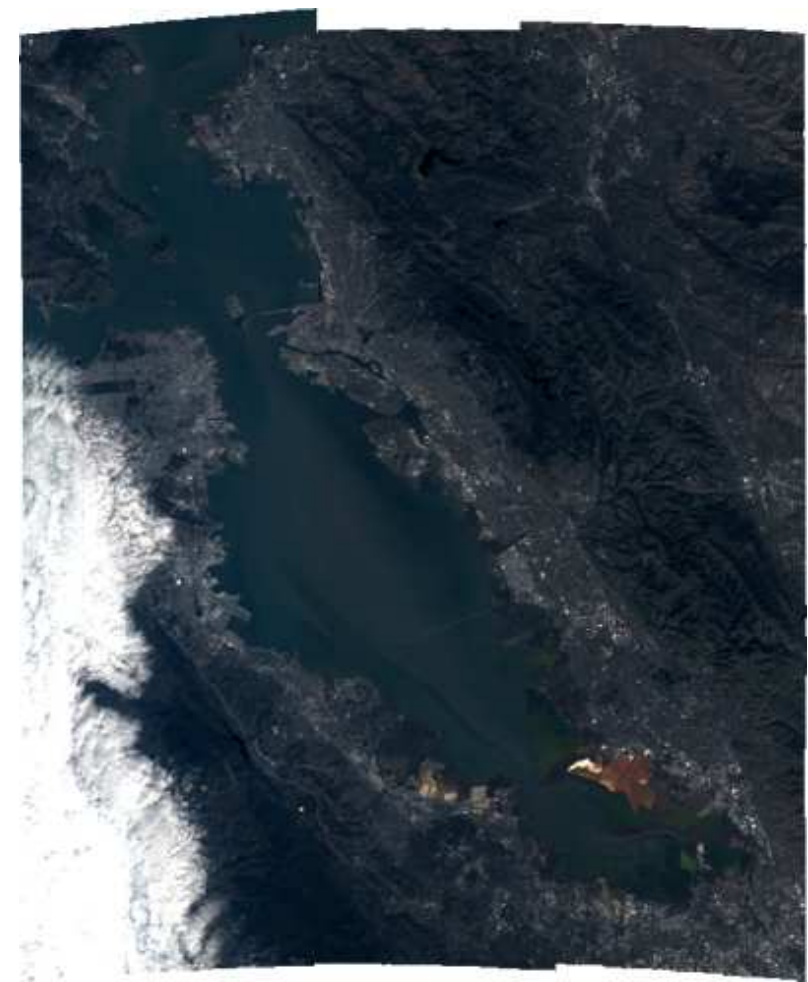

Figure 1.

A Pleaides' 3 strips mosaic of the bay of San Francisco (C)CNES Copyright 2012

\section{MOSAICING PRINCIPLES}

Mosaicing processing is based on three key concepts: refining, harmonizing and unifiing the images.

\subsection{Refine the images to find the common geometry}

First, the refining task consists here in refining the geometric model of the input image sensor [1] [3]. Indeed, each satellite acquisition has its own ground accuracy. This accuracy is given by the precision of the direct model $(\lambda, \phi, h)=F(1, p)$, which gives the ground coordinates $(\lambda, \phi, h)$ for a pixel $(1, p)$ in the image. The precision of this direct function depends very largely on a knowledge of the satellite parameters such as its position and speed (x, y, z, vx, vy, vz), its attitude, (yaw, pitch, roll), the pointing angles and focal plane description, the onboard disturbances (thermal deformations, micro-vibrations) and also the knowledge of the Digital Elevation Model (DEM).

The direct location can be modelised using a precise knowledge of these on-board parameters, but can also be described by a Rational Polynomial Coefficient (RPC). The role of the refining task is to optimize the direct function under constraints to make images fit better with each other.

To do so, the process starts by taking automatically homologous points between images in order to spatio-triangulate the geometric model of all images.

Homologous points can be taken between ground references and images (ground control points, GCPs), between images to be mosaicked themselves (tie points for imagery registration), between spectral bands of an image (multi spectral registration) or between reference images in native geometry and input images. 


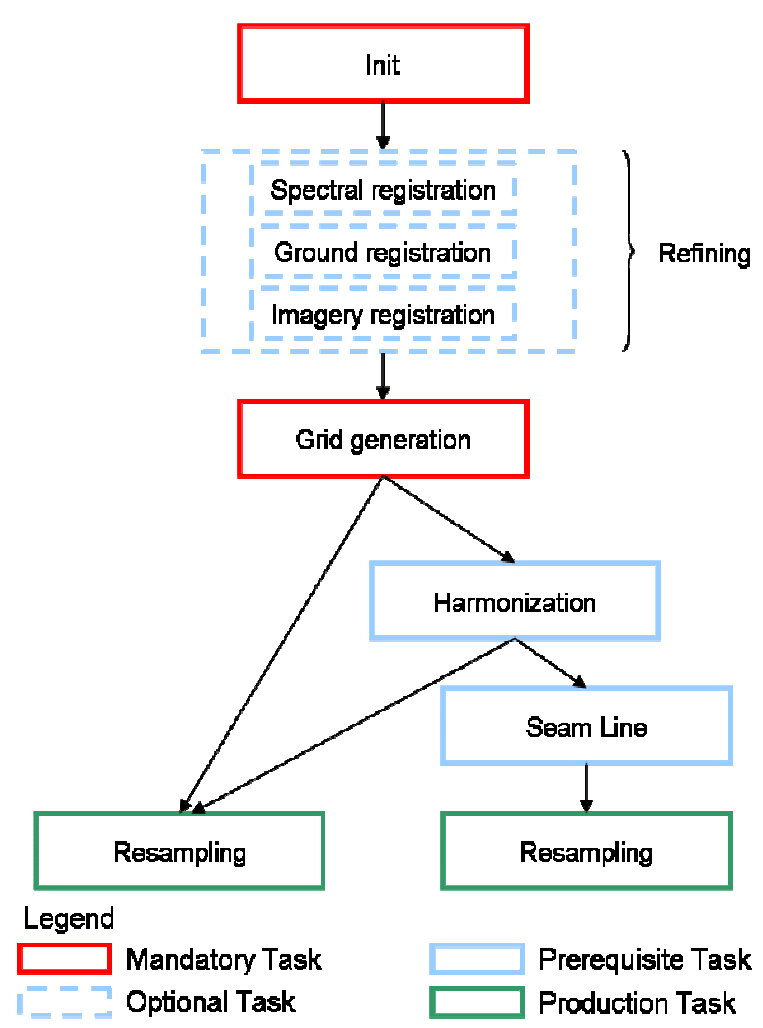

Figure 5. mosaicing processing steps

Homologous points can be detected automatically or provided by the user (not used in operational). If automatic point detection is requested, a preparation phase computes all overlay zones in which the correlation phases are launched. All the images to be registered are re-sampled in a common geometry in order to measure the mis-registration. Homologous points are then chosen by an advanced correlation library, based on pyramidal decomposition, which is highly configurable3. The correlation points are then filtered to establish the list of homologous points.

Finally, all the accumulated homologous points (GCPs, Tie Points etc.) are inserted in a Least Mean Square solver. The residuals provide the correction to be given to each satellite parameter to obtain the new (refined) geometric model of the sensors.

This accurate model is then sampled to obtain sampling grids, which is a way to optimized the processing cost of the resampling step.

\subsection{Harmonize the image to find an homogenized radiometry}

The second main step is the radiometric harmonization. The task involves the computation of a set of Look-Up Table (LUT) grids to ensure harmonization, with particular attention being given to harmonization between images (global harmonization) and harmonization at the overlay zones (local harmonization). Global harmonization is necessary to obtain a satisfactory global impression whereas local harmonization aims at facilitating stitching line computation.

\subsection{Uniting the images with the stitching line}

Thirdly, the stitching line is computed on each overlay zone. For each image pair, a cost map is calculated, based on radiometric difference and gradient difference between images, and then a dynamic programming algorithm is applied to the cost map to detect the least costly line [4]. The cost criteria are optimized to avoid urban constructions and to favor radiometrically homogeneous zones.

The ultimate step for generating orthomosaics is resampling, which takes into account the previously produced resampling grids, the LUT grids and the stitching line. The task can either produce an album resolution mosaic or a full resolution mosaic.

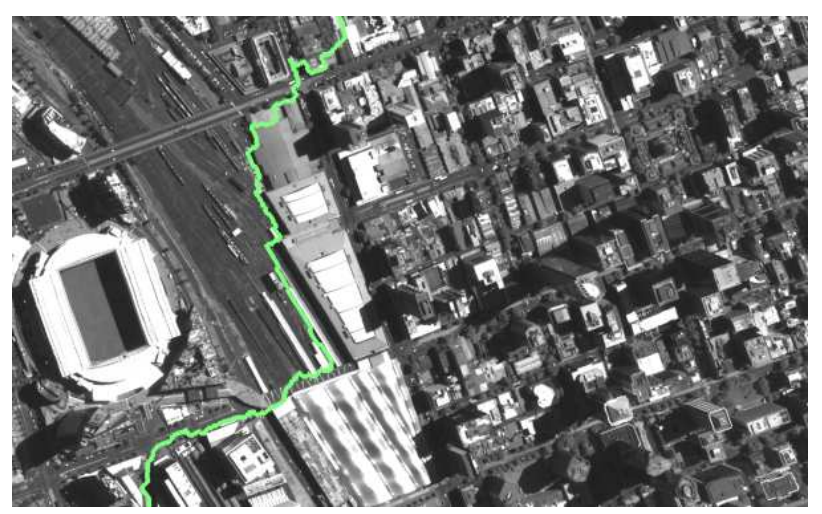

Figure 6. Stiching line : Melbourne stadium CCNES Copyright 2012

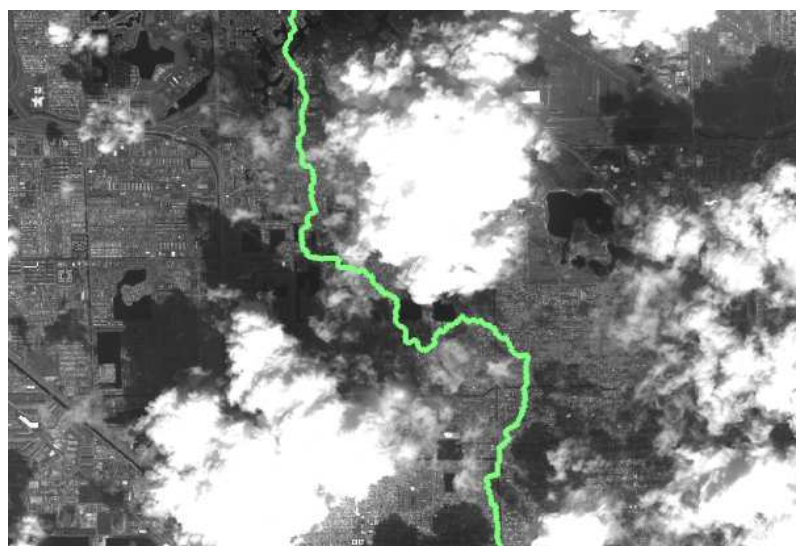

Figure 7. Stiching line avoiding clouds (C)CNES Copyright 2012

\section{MOSAICING UNDER OPERATIONAL CONDITIONS}

\subsection{Making the process to be fully automatic}

An operational requirement was to avoid any human intervention in the Pleiades Image Processing Unit. The mosaicing process is designed to allow mosaic generation without any intervention from the operator. All steps are strung together in a workflow and dataflow manager.

One of the main challenges when creating a mosaic is the control of a large number of steps. For a mosaic using $\mathrm{N}$ images, there can be $\mathrm{N}$ ground control registration tasks, and $\mathrm{N}$ ! tie point registration tasks (if all images have a common zone). Besides, the assembly tree, used for harmonization and stitching 
line computation tasks, can become extremely complex in the event of numerous overlays. All this combinatory complexity has to be hidden from the user, so a planning function to solve all configurations is used.

\subsection{Optimized computing}

The other main operational requirement was to ensure an optimized execution. It thus offers fully automatic execution and time \& memory consumption optimization: all tasks are divided into unitary steps which correspond to unitary processing. Steps that can be executed at the same time are identified, and can be launched in parallel if the mosaicing workflow engine has been assigned to more than one processor.

In operational conditions in the Pleiades Image Processing Unit, more than a hundred of processes can be run in parallel. In test environment, the mosaicing tool can be launched on a single Linux PC by assigning from one to eight processors on a octocore configurations.

\section{CONCLUSION}

Designed and developed for use in the Pleiades operational Image Processing Unit, SIGMA is an automatic and generic integrated mosaicing system. It has already shown complete satisfaction in integration phases, regarding both image quality and performance. It is currently being tuned during Pleiades inorbit commissioning phase but has already provided outstanding results. SIGMA is also used for other CNES or European projects where CNES contribute [5].

\section{REFERENCES}

[1] F. De Lussy et al., "Pleiades-HR system products and quality, Pleiades-HR system products and geometric accuracy", ISPRS Hannover Workshop, 2005.

[2] H. Le Men and D. Boldo, "Mosaïque automatique d'orthophotographies", RFIA'2000, laboratoire MATIS, IGNSR 990

[3] S. Baillarin, P. Gigord, O. Hagolle, "Automatic Registration of Optical Images, a Stake for Future Missions: Application to Ortho-Rectification, Time Series and Mosaic Products", IGARSS 2008

[4] J. Chon, H. Kim "Determination of the Optimal Seam-Lines in Image Mosaicking with the Dynamic Programming (DP) on the Converted Cost Space", ICAISC 2006

[5] Aimé Meygret et al. "Sentinel-2 Image Quality and Level 1 Processing”, SPIE San Diego, 2010

\section{ACKNOWLEDGEMENTS}

We would like to thank all our colleagues from CNES Pleiades project team. We are also grateful to Yannick Ott, Daphne Deleflie, Christine Botella from Thales Services and Jean-Louis Dallaverde, Frederic Claudel, Laurent David from Magellium and their teams for their highly valuable work on the SIGMA and Image Processing chain development.

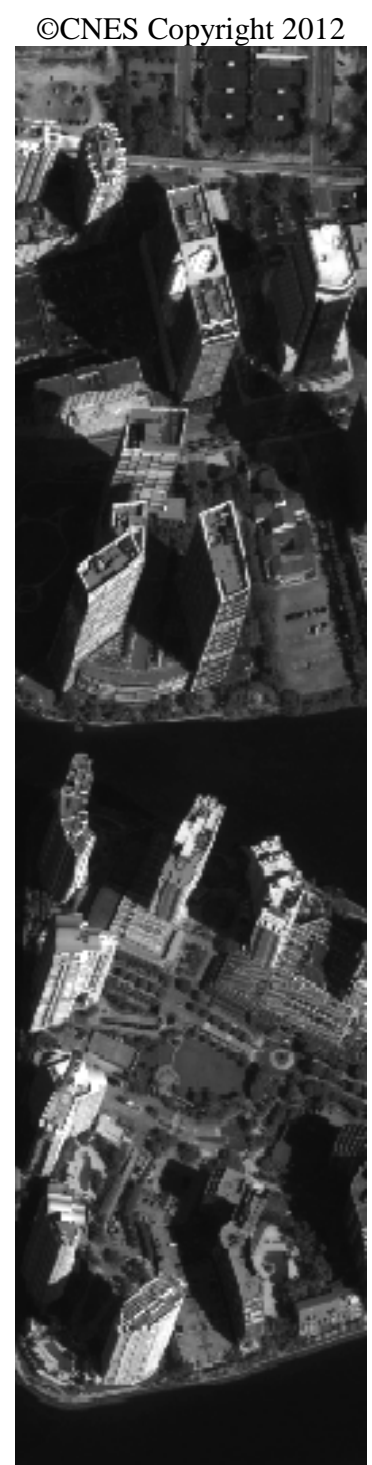

